\title{
METTL3 Attenuates LPS-Induced Inflammatory Response in Macrophages via NF- $\kappa$ B Signaling Pathway
}

\author{
Jinghua Wang, ${ }^{1,2}$ Shushan Yan $\mathbb{D}^{3}$ Hongying Lu, ${ }^{4}$ Shufeng Wang $\mathbb{D}^{5}$ and Donghua Xu $\mathbb{D}^{1,2}$ \\ ${ }^{1}$ Clinical Medicine College, Weifang Medical University, Weifang 261000, China \\ ${ }^{2}$ Department of Rheumatology, The Affiliated Hospital of Weifang Medical University, Weifang 261000, China \\ ${ }^{3}$ Department of Gastrointestinal and Anal Diseases Surgery, The Affiliated Hospital of Weifang Medical University, \\ Weifang 261000, China \\ ${ }^{4}$ Functional Laboratory, Clinical Medicine College, Weifang Medical University, Weifang 261000, China \\ ${ }^{5}$ Medical Experimental Training Center, Weifang Medical University, Weifang 261000, China
}

Correspondence should be addressed to Shushan Yan; yanshushan@163.com, Shufeng Wang; wfwangshufeng@163.com, and Donghua Xu; flower322@163.com

Received 20 March 2019; Accepted 5 September 2019; Published 24 October 2019

Academic Editor: Daniela Caccamo

Copyright ( 2019 Jinghua Wang et al. This is an open access article distributed under the Creative Commons Attribution License, which permits unrestricted use, distribution, and reproduction in any medium, provided the original work is properly cited.

Methyltransferase-like 3 (METTL3), an RNA $\mathrm{N}^{6}$-methyladenosine $\left(\mathrm{m}^{6} \mathrm{~A}\right)$ methyltransferase, is essential for the $\mathrm{m}^{6} \mathrm{~A} m \mathrm{~m} \mathrm{NA}$ modification. As a key enzyme of $\mathrm{m}^{6} \mathrm{~A}$ methylation modification, METTL3 has been implicated in immune and inflammation regulation. However, little is known of the role and underlying mechanism of METTL3 in rheumatoid arthritis (RA). The aim of the present study is to elucidate the function and potential mechanism of METTL3 in RA pathogenesis. We used quantitative real-time polymerase chain reaction to detect the expression of METTL3 in RA patients and controls as well as the macrophage cell line. CCK-8 was used for cell proliferation assay. Enzyme-linked immunosorbent assay (ELISA) was adopted to estimate the generation of IL- 6 and TNF- $\alpha$ in macrophages. Western blot and immunofluorescence were applied to evaluate the activation of NF- $\kappa$ B in macrophages. The expression of METTL3 was significantly elevated in patients with RA. It was positively associated with CRP and ESR, two common markers for RA disease activity. Besides, LPS could enhance the expression and biological activity of METTL3 in macrophages, while overexpression of METTL3 significantly attenuated the inflammatory response induced by LPS in macrophages. Moreover, the effect of METTL3 on LPS-induced inflammation in macrophages was dependent on NF- $\kappa$ B. This study firstly demonstrates the critical role of METTL3 in RA, which provides novel insights into recognizing the pathogenesis of RA and a promising biomarker for RA.

\section{Introduction}

Rheumatoid arthritis (RA) is an autoimmune disease with chronic and reduplicated joint destruction, which is a highly disabling disease because of joint deformity and loss of function [1]. Joint inflammation can cause redness, swelling, pain, and joint deformity [2]. The etiology of RA is still largely unknown. It has been well established that the development of RA is attributed to genetic and environmental factors, such as tobacco smoking [3]. Apart from those, obesity, stress, nervous depression, and female hormones play vital roles in the pathogenesis of RA [3]. Many studies have suggested that dysregulation of the immune system, includ- ing abnormal activation and $\mathrm{T}$ and $\mathrm{B}$ lymphocytes, mast cells, and macrophages, is closely associated with the development of RA [4]. High levels of autoantibodies generated by dysregulated B cells can cause lung injuries, such as anticitrullinated protein antibodies $[5,6]$. Inflammatory cells can be attracted and recruited to inflammatory sites under stimulation of considerable mediators from macrophages and mast cells. Thus, sustained and chronic inflammation leads to joint injuries and deformity [1]. As a result, it is essential to identify novel and promising biomarkers for the early diagnosis and targeted therapy of RA patients.

During the past few years, the role of $\mathrm{N}^{6}$-methyladenosine $\left(\mathrm{m}^{6} \mathrm{~A}\right)$ methylation of RNA in autoimmunity, inflammation, 
TABLE 1: Characteristics of participants.

\begin{tabular}{lccc}
\hline & RA & Healthy & P value \\
& $(n=47)$ & controls $(n=30)$ & \\
\hline Age (yrs, mean \pm SD) & $58.3 \pm 6.1$ & $49.9 \pm 8.7$ & 0.242 \\
Gender (females/males) & $34 / 13$ & $21 / 9$ & 0.105 \\
Disease course (years) & $16.6 \pm 5.2$ & - & - \\
CRP $(\mathrm{mg} / \mathrm{l})$ & $20.4 \pm 3.5$ & - & - \\
ESR $(\mathrm{mm} / \mathrm{h})$ & $41.0 \pm 7.5$ & - & - \\
RF $(\mathrm{IU} / \mathrm{ml})$ & $191.6 \pm 5.7$ & - & - \\
\hline
\end{tabular}

and cancer has drawn close attention [7-10]. $\mathrm{m}^{6} \mathrm{~A}$ is the most common posttranscription modification of RNA [11]. The key enzymes for $\mathrm{m}^{6} \mathrm{~A}$ methylation modification primarily include $\mathrm{m}^{6} \mathrm{~A}$ methyltransferase (writer), $\mathrm{m}^{6} \mathrm{~A}$ demethylase (eraser), and $\mathrm{m}^{6} \mathrm{~A}$ RNA-binding proteins (reader) [12]. Methyltransferase-like 3 (METTL3) is a key enzyme of $\mathrm{m}^{6} \mathrm{~A}$ methylation modification and an important member of the methyltransferase complex including METTL3, METTL4, and Wilms tumor 1-associated protein (WTAP) [13]. It has been demonstrated that $\mathrm{m}^{6} \mathrm{~A}$ methylation mediated by METTL3 has tissue and cell specificity [12-15]. Therefore, the role of METTL3 may alter in different tissues and cells [16]. METTL3 has been previously reported as a tumor suppressor by upregulating the m6A modification of genes [17]. However, Chen and colleagues have found that METTL3 could promote liver cancer progression by posttranscriptional silencing of SOCS2 via YTHDF2 [18]. More interestingly, the study by Feng et al. has implicated that METTL3 could inhibit inflammation by affecting the alternative splicing of MyD88 [19]. Nevertheless, the role of METTL3 in RA, an autoimmune and inflammatory disease, remains vague up to date. In the current study, we investigate the expression of METTL3 in RA and its relationship with disease activity. A series of cellular experiments in vitro have been performed to elucidate the potential role of METTL3 and its molecular mechanisms in RA, which may help to explore the pathogenesis of RA and explore novel biomarkers for RA.

\section{Materials and Methods}

2.1. Participants. Patients (47) who participated in this study were admitted to the affiliated hospital of Weifang Medical University from March 2018 to September 2018. 30 controls registered in the same hospital at the same time for health examination, while controls with a history of rheumatoid diseases were all excluded. All RA cases were new active patients and had not yet been treated with diseasemodifying antirheumatic drugs and/or steroids before blood sample collection. Those RA patients accompanied by other rheumatoid diseases were excluded, such as Sjogren's disease. The present study was approved by the Institutional Ethics Committee of our hospital. Patients and controls had all signed the written informed consent before tests. Characteristics of all participants are presented in detail in Table 1.
2.2. Cell Culture and Transfection. THP-1 cells were cultured in RPMI1640 culture medium (Invitrogen Corp., Grand Island, NYUSA) administrated with $10 \%$ fetal bovine serum (Gibco, Carlsbad, CA, USA) plus penicillin, streptomycin, and L-glutamine under $5 \% \mathrm{CO}_{2}$ at $37^{\circ} \mathrm{C}$. Before treatment, THP- 1 cells were stimulated and induced to macrophagelike cells (pTHP-1) by $100 \mathrm{ng} / \mathrm{ml}$ phorbol-12-myristate- 13 acetate (PMA, Sigma, USA) for $48 \mathrm{~h}$. Cells were transfected by lentivirus plasmid with polybrene $(8 \mu \mathrm{g} / \mathrm{ml})$ for $48 \mathrm{~h}$ and used for the following experiments.

\subsection{Quantitative Real-Time Polymerase Chain Reaction} ( $q R T-P C R)$. Peripheral blood mononuclear cells (PBMCs) were extracted from fresh blood samples by Ficoll-Paque lymphocyte isolate reagent (TBD, Tianjin, China), which were centrifuged at $2000 \mathrm{rpm}$ for $30 \mathrm{~min}$. We extracted peripheral blood monocytes using CD14 microbeads (Miltenyi Biotec, San Diego, CA) based on the protocol. Total RNAs were extracted from these cells by TRIzol (Invitrogen, USA) and quantified by measuring the absorbance with the UV spectrophotometer. A total of $1 \mu \mathrm{g}$ RNA was used to synthesize cDNA, which was used as a template for PCR by use of a SYBR Green Mastermix kit (Takara, Dalian, China). Primers were shown as follows. GAPDH: forward primer: GCACCGTCAAGGCTGAGAAC; reverse primer: GGAT CTCGCTCCTGGAAGATG. METTL3: forward primer: TTGTCTCCAACCTTCCGTAGT; reverse primer: CCAG ATCAGAGAGGTGGTGTAG. METTL14: forward primer: AGTGCCGACAGCATTGGTG; reverse primer: GGAGCA GAGGTATCATAGGAAGC. Obesity-associated protein (FTO): forward primer: AGAGCTCTAGAACCACCATGG ATTACAAAGATGAC; reverse primer: CTAAGATTGCG GCCGCCTAGGGTTTTGCTTCCAGAAGC. AlkB homologue 5 (ALKBH5): forward primer: CGGCGAAGGCT ACACTTACG; reverse primer: CCACCAGCTTTTGGAT CACCA. YTHGF1: forward primer: ACCTGTCCAGCTAT TACCCG; reverse primer: TGGTGAGGTATGGAATCGG AG. YTHGF2: forward primer: AGCCCCACTTCCTACC AGATG; reverse primer: TGAGAACTGTTATTTCCCCAT GC. IL-6: forward primer: AGTCCTGATCCAGTTCCTGC; reverse primer: CTACATTTGCCGAAGAGCCC. TNF- $\alpha$ : forward primer: ATGTGGCAAGAGATGGGGAA; reverse primer: CTCACACCCCACATCTGTCT.

2.4. Total $m^{6} A$ Measurement. Cells were seeded in six-well culture dishes overnight in serum-free RPMI1640 culture medium. Then, cells were treated with $1 \mu \mathrm{g} / \mathrm{ml}$ LPS (Sigma, CA, USA) for $0,6,12$, and $24 \mathrm{hrs}$. RNAs were extracted according to the protocol. The kit for methylation quantification (EpiGentek, Farmingdale, NY, USA) was applied to determine levels of $\mathrm{m}^{6} \mathrm{~A}$ RNA modification in cells.

2.5. Cell Counting Kit (CCK-8). We used CCK-8 kit (Vazyme Biotech, Nanjing, China) to determine cell proliferation. In brief, $2 \times 10^{4}$ per well THP-1 cells were incubated and activated by PMA $(100 \mathrm{ng} / \mathrm{ml})$ for $48 \mathrm{hrs}$ in a 96-well plate and cultured with serum-free culture medium for $12 \mathrm{hrs}$ and then stimulated by $1 \mu \mathrm{g} / \mathrm{ml}$ LPS (Sigma, USA) for $0,12,24$, and $48 \mathrm{hrs}$. The absorption at $450 \mathrm{~nm}$ was determined by the 


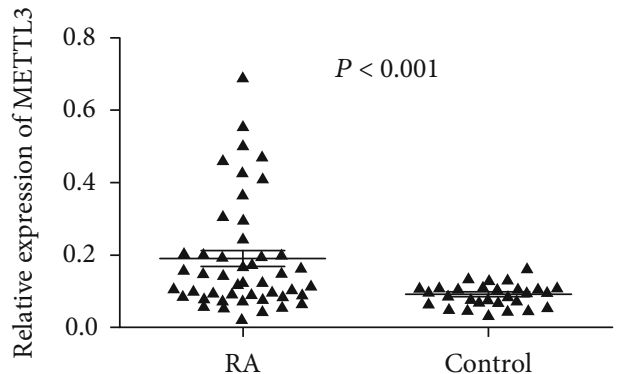

(a)

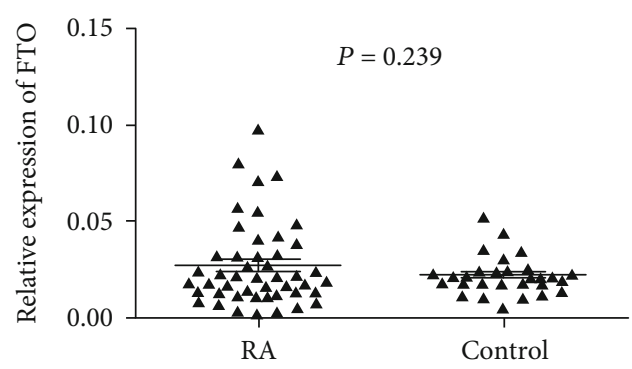

(c)

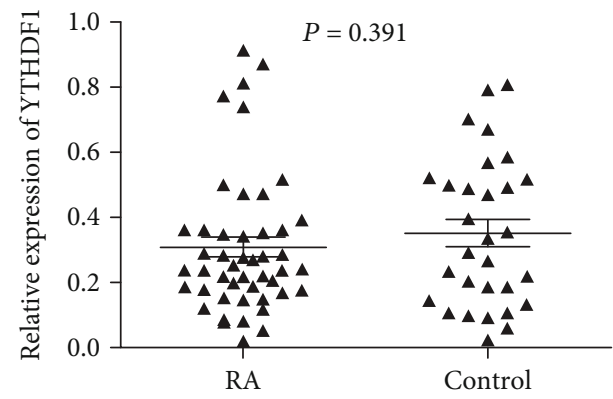

(e)

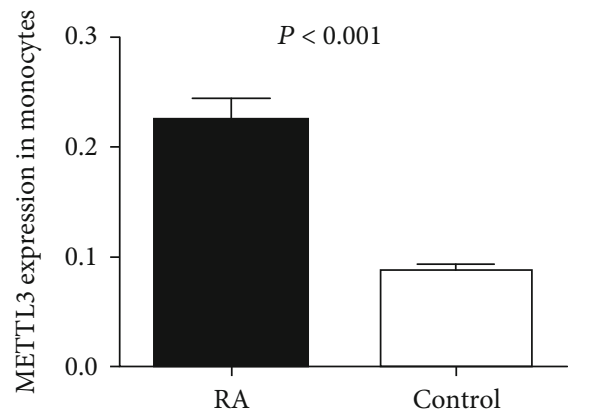

(g)

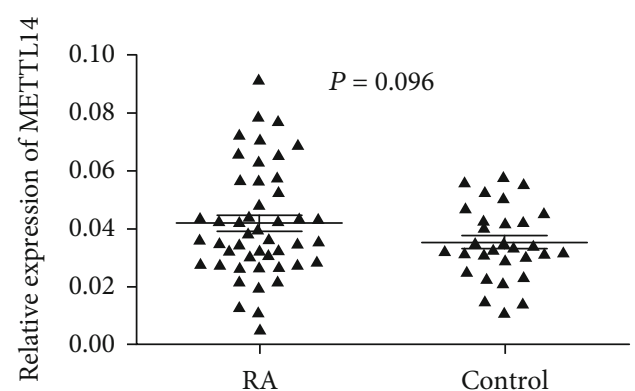

(b)

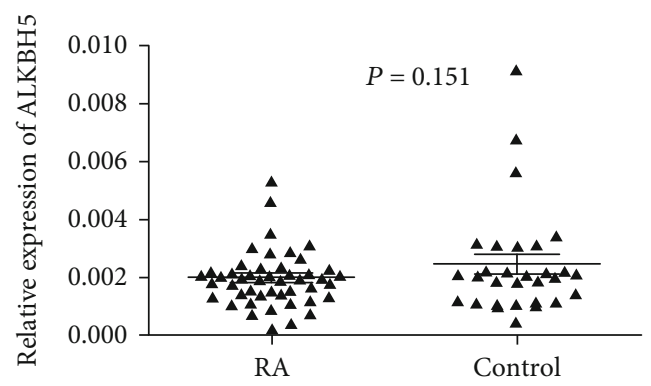

(d)

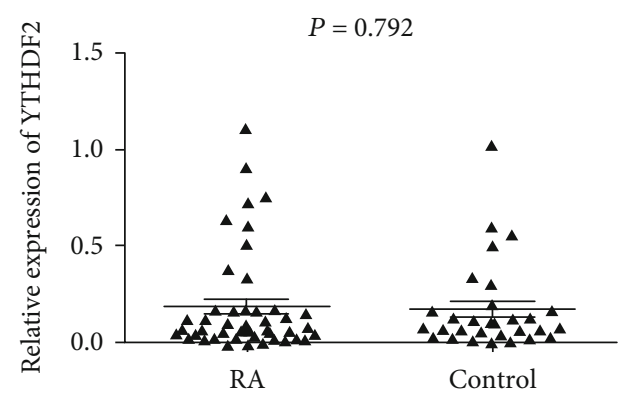

(f)

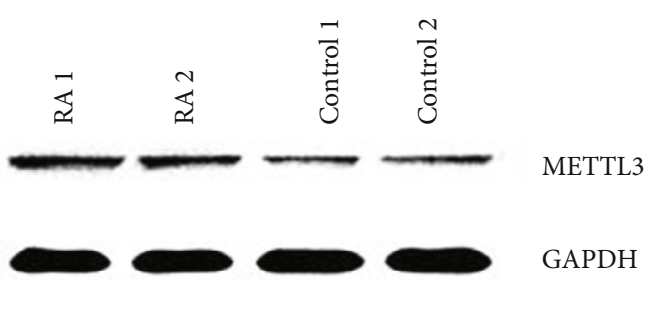

(h)

FIGURE 1: Expression of $\mathrm{m}^{6} \mathrm{~A}$ methylation-related genes in RA (cases/controls: 47/30). (a) Increased mRNA level of METTL3 in RA in contrast to healthy controls. (b) mRNA level of METTL14 in RA when comparing with healthy controls. (c) mRNA level of FTO in RA compared with healthy controls. (d) mRNA level of ALKBH5 in RA in contrast to healthy controls. (e) mRNA level of YTHDF1 in RA when comparing with healthy controls. (f) mRNA level of YTHDF2 in RA in contrast to healthy controls. (g) Increased mRNA level of METTL3 in monocytes of RA patients in contrast to controls. (h) Increased METTL3 protein in monocytes of RA patients in contrast to controls.

microplate reader (BioTek, USA) after incubation with CCK8 reagent for $2 \mathrm{hrs}$ at $37^{\circ} \mathrm{C}$.

2.6. Enzyme-Linked Immunosorbent Assay (ELISA). The concentrations of IL-6, TNF- $\alpha$, ESR, and CRP were determined by ELISA according to the kit's protocol (R\&D Systems, USA). We determined the absorption at the wavelength of
$450 \mathrm{~nm}$ with a correction wavelength of $540 \mathrm{~nm}$. Experiments were repeated for three times.

2.7. Western Blot. A total of $30 \mu \mathrm{g} / \mathrm{ch}$ annel proteins were used for detection extracted from peripheral blood monocytes and pTHP-1 cells. We adopted the Bradford assay kit (Bio-Rad Laboratories, CA, USA) to quantify proteins. Protein samples 


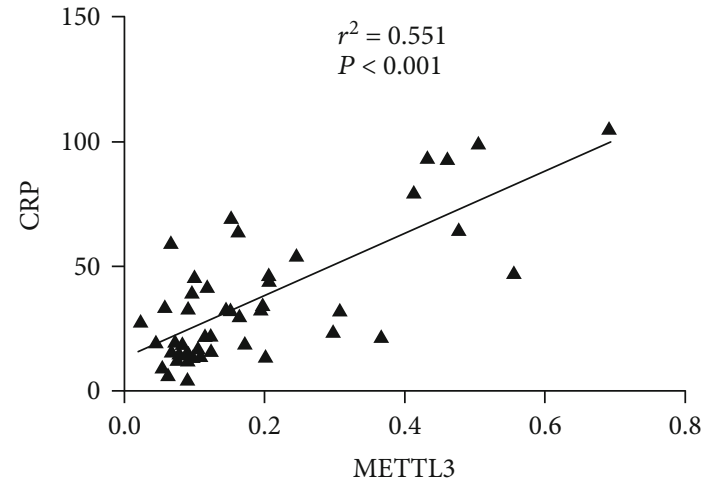

(a)

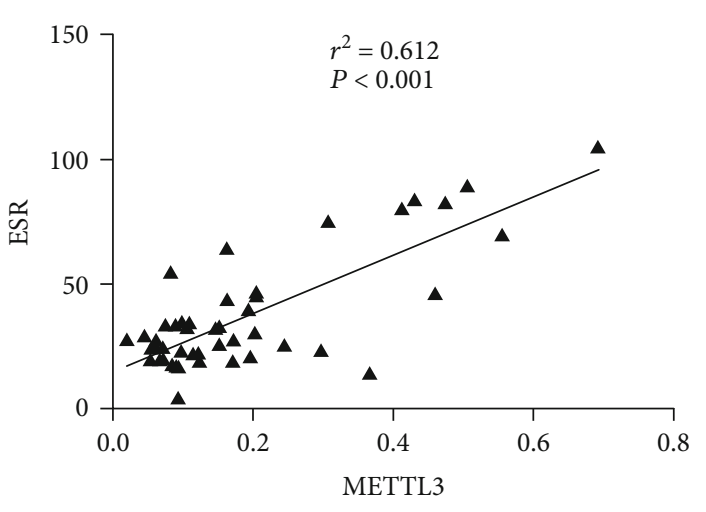

(b)

Figure 2: Association between METTL3 and RA disease activity. (a) Positive association of METTL3 with CRP in RA. (b) Positive association of METTL3 with ESR in RA.

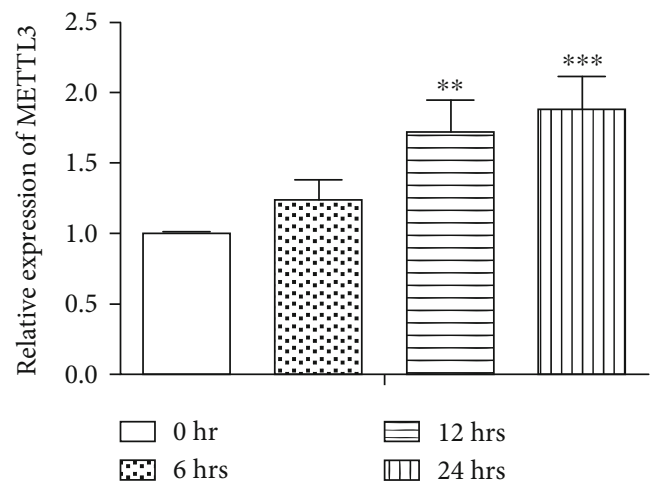

(a)

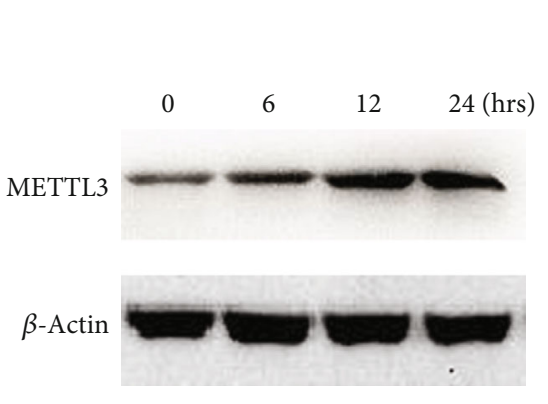

(b)

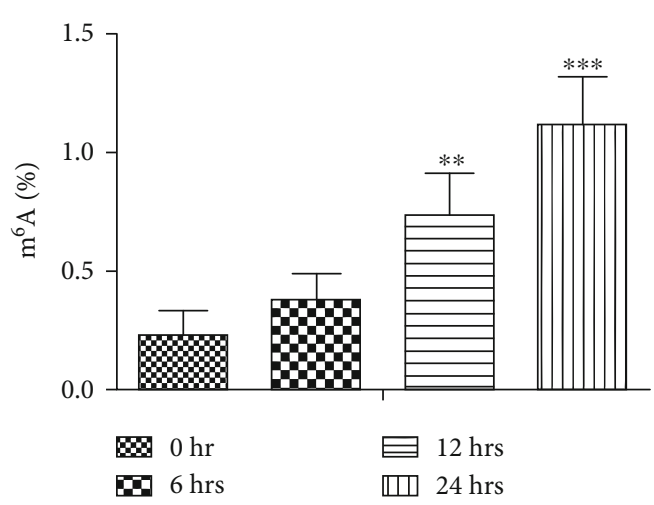

(c)

FIGURE 3: LPS promoted the expression and activity of METTL3 in pTHP-1 cells. (a) Increased mRNA level of METTL3 in cells ( $n=3$; LPS stimulation for $0 \mathrm{hr}, 6 \mathrm{hrs}, 12 \mathrm{hrs}$, and $24 \mathrm{hrs} ;{ }^{* *} P<0.01$ and $\left.{ }^{* * *} P<0.001\right)$. (b) Increased expression of METTL3 protein in cells $(n=3$; LPS stimulation for $0 \mathrm{hr}, 6 \mathrm{hrs}, 12 \mathrm{hrs}$, and $24 \mathrm{hrs}$ ). (c) Total $\mathrm{m}^{6} \mathrm{~A}$ content in cells (LPS stimulation for $0 \mathrm{hr}, 6 \mathrm{hrs}, 12 \mathrm{hrs}$, and $24 \mathrm{hrs}$; $n=3$; ${ }^{* *} P<0.01$ and $\left.{ }^{* * *} P<0.001\right)$.

were incubated with antibodies of METTL3 (Cell Signaling Technology, USA) and p-NF- $\kappa$ B (Cell Signaling Technology, USA), and $\beta$-actin (Sigma, USA).

2.8. Immunofluorescence. After being activated by LPS $(1 \mu \mathrm{g} / \mathrm{ml})$ for $4 \mathrm{hrs}$, the status of phosphorylated NF- $\kappa \mathrm{B}$ in the nuclear of pTHP-1 cells was estimated by immunofluorescence. Briefly, pTHP-1 cells were incubated with p-NF- $\kappa$ B antibody (CST, USA) and then the secondary antibodies labelled with FITC. Finally, cells were detected using a Confocal Laser Scanning Microscope (Leica TCS SP8).

2.9. Statistical Analysis. Statistical analysis was carried out by use of GraphPad Prism (GraphPad Software, CA, USA). Data used for analysis was normal distributed. Unpaired Student's $t$-test or one-way ANOVA was used for statistical analysis. 


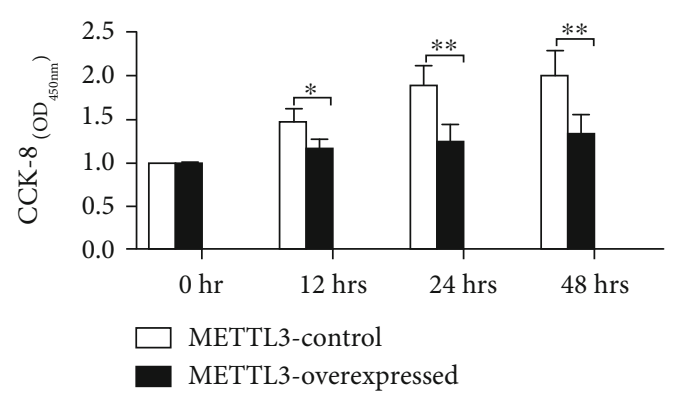

(a)

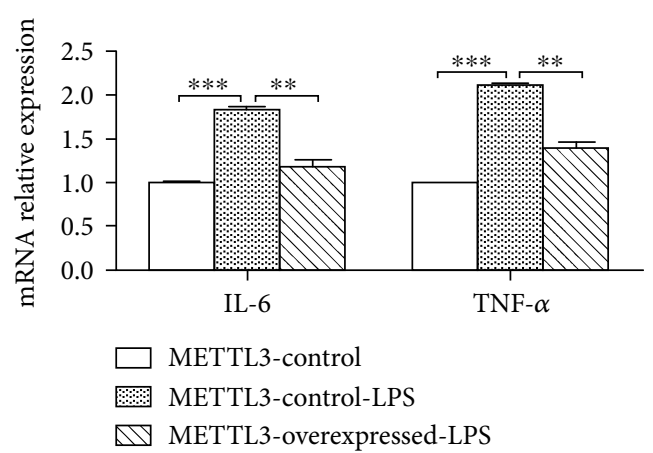

(b)

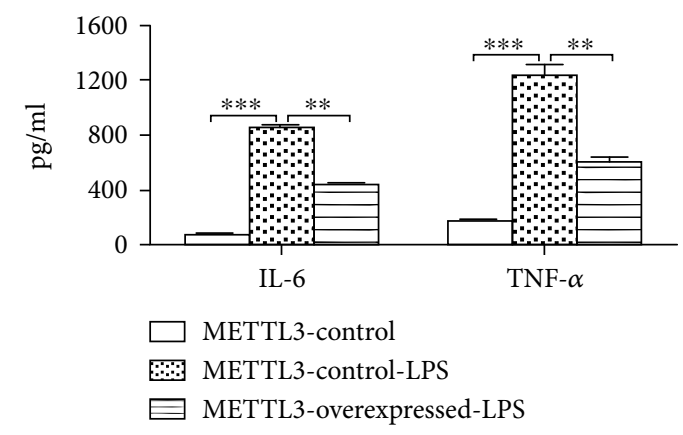

(c)

FIGURE 4: METTL3 inhibited the proliferation and activation of pTHP-1 cells. (a) CCK- 8 assay detecting the proliferation of cells at 0 hr, $12 \mathrm{hrs}, 24 \mathrm{hrs}$, and $48 \mathrm{hrs}\left(n=3{ }^{*} P<0.05\right.$; $\left.{ }^{* *} P<0.01\right)$. (b) Decreased mRNA levels of IL- 6 and TNF- $\alpha$ in METTL3-overexpressed pTHP-1 cells $\left(n=3 ;{ }^{* *} P<0.01 ;{ }^{* *} P<0.001\right)$. (c) Decreased levels of IL-6 and TNF- $\alpha$ protein in the cultural supernatant of METTL3overexpressed cells $\left(n=3{ }^{* *} P<0.01\right.$ and $\left.{ }^{* * *} P<0.001\right)$.

Pearson correlation analysis was used when analyzing the association between the expression of METTL3 and levels of ESR and CRP in serum. Two-tailed $P<0.05$ was regarded to be statistically significant.

\section{Results}

3.1. Expression of RNA N6 Methylation-Related Genes in RA. We screened the expression of $\mathrm{m}^{6} \mathrm{~A}$ methylation-related genes in the PBMCs of patients with RA and healthy controls by quantitative real-time PCR, including genes of METTL3, METTL14, FTO, ALKBH5, YTHDF1, and YTHDF2. As shown in Figure 1(a)-1(f), compared with normal controls, the expression of METTL3 was significantly increased in PBMCs from RA patients, while no difference was observed with regard to other key $\mathrm{m}^{6} \mathrm{~A}$ methylation-related enzymes (METTL14, FTO, ALKBH5, YTHDF1, and YTHDF2). Monocytes were the main cells involved in inflammation and immune regulations. Here, elevated expression of METTL3 was also found in monocytes of RA patients in contrast to controls (Figures $1(\mathrm{~g})$ and $1(\mathrm{~h})$ ). Taken together, METTL3 was upregulated in RA.

3.2. Association between METTL3 and Disease Activity of RA Patients. Interestingly, Pearson correlation analysis showed that the expression of METTL3 was positively associated with CRP (Figure 2(a)). Similarly, positive association of the expression of METTL3 with ESR was observed in RA
(Figure 2(b)). Accordingly, the elevated level of METTL3 in PBMCs might predict high disease activity of patients with RA.

3.3. LPS Enhanced the Expression of METTL3. Given the positive association between METTL3 and CRP as well as ESR in RA, we hypothesized that a high level of METTL3 could be induced in inflammatory conditions, which could thus defend against inflammation. As a result, we performed cellular experiments in vitro to observe the influence of inflammation on METTL3 expression and the level of $\mathrm{m}^{6} \mathrm{~A}$ RNA modification in pTHP-1 macrophages. LPS could enhance the expression of METTL3 at both levels of mRNA and protein in a time-dependent manner (Figures 3(a) and 3(b)). Levels of $\mathrm{m}^{6} \mathrm{~A}$ RNA modification were also increased in pTHP-1 macrophages in a time-dependent manner (Figure 3(c)). Taken together, inflammation could promote the expression and biological activity of METTL3. However, whether METTL3 affected inflammation in macrophages and RA development remained unknown.

3.4. METTL3 Inhibited the Activation of pTHP-1 Macrophages. As evidenced by the CCK- 8 assay, the proliferation of macrophages was significantly inhibited in METTL3-overexpressed pTHP-1 cells after being activated by LPS for 12, 24, and 48 hrs (Figure 4(a)). In addition, the generation of IL- 6 and TNF- $\alpha$ induced by LPS was obviously prevented when METTL3 was overexpressed in pTHP-1 


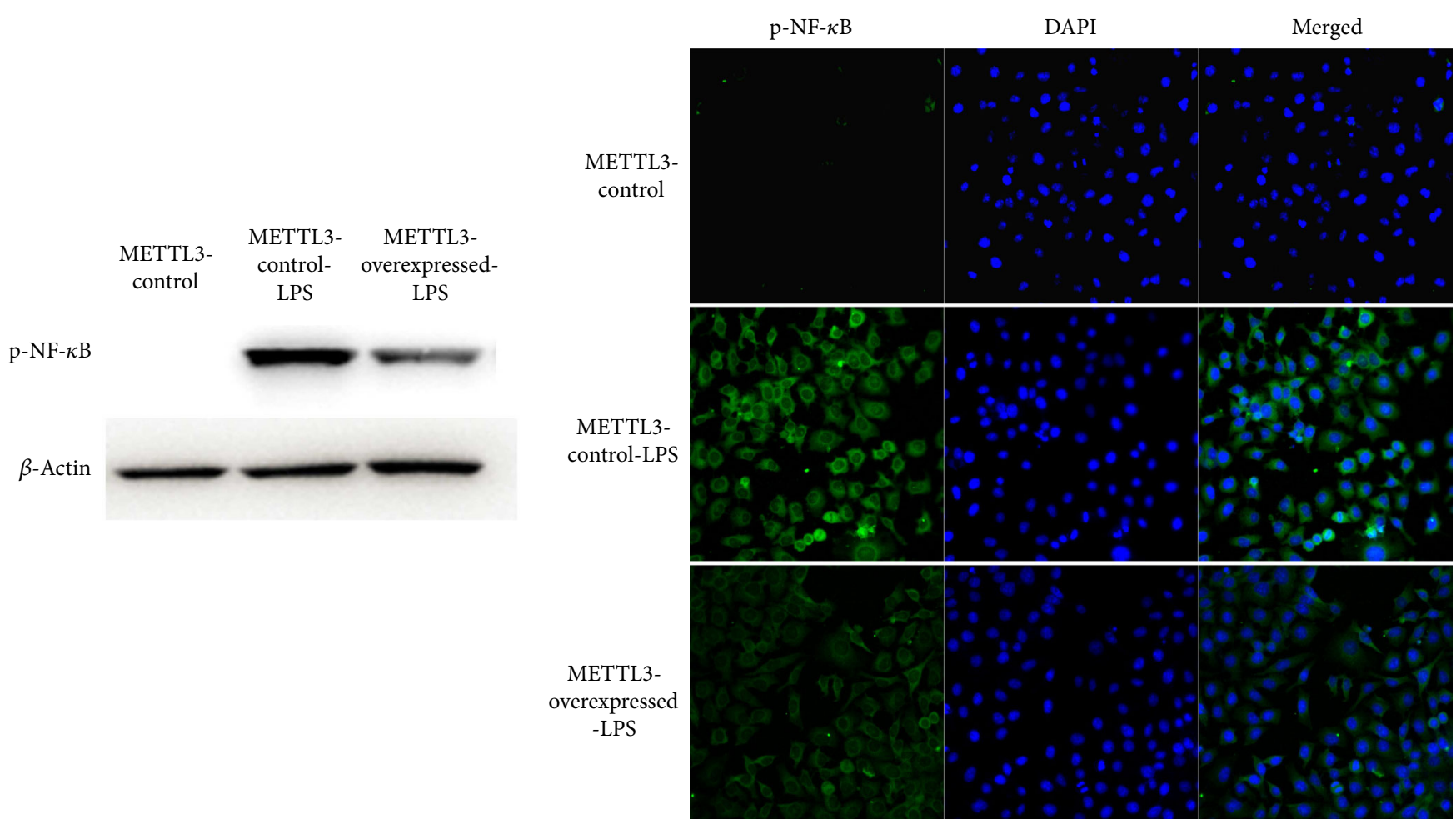

(a)

(b)

FIGURE 5: METTL3 attenuated LPS-induced inflammation in macrophages through the NF- $\kappa$ B signaling pathway. (a) METTL3 inhibited the phosphorylation of NF- $\kappa$ B in cells induced by LPS $(n=3)$. (b) METTL3 inhibited the nucleus translocation of $p-N F-\kappa B$ in cells induced by LPS $(n=3)$.

macrophages (Figures 4(b) and 4(c)). Accordingly, as a key enzyme of $\mathrm{N}^{6}$-methyladenosine $\left(\mathrm{m}^{6} \mathrm{~A}\right)$ methylation, METTL3 could affect RA by inhibiting the proliferation and inflammatory response in macrophages, which played a crucial role in RA.

3.5. METTL3 Inhibited the Inflammatory Response of pTHP-1 Macrophages through NF- $\kappa B$. Dysregulation of METTL3 seemed to influence the inflammation response of macrophages. Nonetheless, the potential molecular mechanism remained vague. In this study, we found that the phosphorylation of $\mathrm{NF}-\kappa \mathrm{B}$ was obviously restrained in METTL3-overexpressed pTHP-1 cells although cells were activated by LPS for $4 \mathrm{hrs}$ (Figure 5(a)). Besides, the nuclear translocation of phosphorylated NF- $\kappa \mathrm{B}$ in cells was also restrained when METTL3 was overexpressed (Figure 5(b)). Taken together, the modifying effects of METTL3 on LPSinduced inflammation in macrophages were dependent on the transcriptional factor $\mathrm{NF}-\kappa \mathrm{B}$.

\section{Discussion}

$\mathrm{RA}$ is a chronic and systemic autoimmune disease, the etiology of which is largely unknown [20]. It has been well documented that multiple factors are associated with inflammation and immune disorders in RA, including genetic factors, environmental factors, and epigenetic deregulation $[21,22]$. Various inflammatory mediators are responsible for arthritis and articular deformity, such as TNF- $\alpha$, IL-6, and IL-17 [23]. Apart from inflammation, autoimmune disorders are closely related to the development and progression of RA. As a key enzyme of $\mathrm{m}^{6} \mathrm{~A}$ methylation modification, METTL3 has been suggested to regulate inflammation and autoimmune balance $[13,19]$. The present study firstly shows the evidence that METTL3 is upregulated in RA and positively associated with the disease activity. Besides, METTL3 inhibits the proliferation and activation of macrophages through NF- $\kappa \mathrm{B}$.

$\mathrm{m}^{6} \mathrm{~A}$ is a methylation at the $\mathrm{N} 6$ position of adenosine, which is regarded as the most abundant epitranscriptomic modification of mRNA in eukaryotic cells [24]. A number of studies have demonstrated that $\mathrm{m}^{6} \mathrm{~A}$ methylation is involved in various physiological processes including mRNA stability and translation. Its modifying effects on embryonic development, cell differentiation, and stress have been well demonstrated [24-26]. There are several key genes involved in $\mathrm{m}^{6} \mathrm{~A}$ methylation modification, primarily including METTL3, METTL14, FTO, ALKBH5, and YTHDF [27, 28]. METTL3 is also known as MTA70, which is originally identified as a methyltransferase involved in the process of $\mathrm{m}^{6} \mathrm{~A}$ methylation [29]. Accumulated evidence has strongly supported that METTL3 is involved in a variety of physiological contexts and in cancers [30-32]. METTL3 is located in a nuclear speckle, which reveals that METTL3 may play an important role in RNA metabolism $[33,34]$. Given its effects on inflammation, cancer, and immune regulation [19], we hypothesize that METTL3 may affect the development and progression of RA by regulating 
macrophage-mediated inflammation. In this study, we firstly detected the expression of $\mathrm{m}^{6} \mathrm{~A}$ methylation-associated genes (METTL3, FTO, ALKBH5, METTL14, YTHDF1, and YTHDF2) in PBMCs from RA patients and found that METTL3 was obviously upregulated in RA compared with healthy controls. Interestingly, LPS stimulation could enhance total $\mathrm{m}^{6} \mathrm{~A}$ content of macrophages in a timedependent manner by upregulating METTL3. It can be concluded that METTL3 is upregulated in macrophages under the circumstances of inflammation.

Macrophages are activated when they are infected by pathogenic factors such as LPS, PGN, and nucleotide compositions of pathogenic microorganisms, which leads to the phosphorylation and translocation of the transcriptional factor $\mathrm{NF}-\kappa \mathrm{B}$ to the nucleus and induces the generation of target genes related to inflammation, for instance, IL- 6 and TNF- $\alpha[33,35,36]$. The NF- $\kappa \mathrm{B}$ signaling pathway is a classic pathway related to inflammation and immune regulation in RA [37]. Growing data showed that expression and regulation of $\mathrm{m}^{6} \mathrm{~A}$ methylation-related genes are associated with a variety of immune signaling pathways, particularly the $\mathrm{NF}-\kappa \mathrm{B}$ pathway $[8,18]$. In this study, METTL3 has been found to be upregulated in peripheral blood monocytes, the critical cell type in PMBCs involved in inflammation and immune regulations. In addition, we have demonstrated the effect of METTL3 on $\mathrm{m}^{6} \mathrm{~A}$ modification in macrophages stimulated by LPS. As a result, we hypothesize that METTL3-dependent $\mathrm{m}^{6} \mathrm{~A}$ modification is associated with inflammation induced by LPS in macrophages. After verification in experiments in vitro, we have found that METTL3 could prevent macrophages from proliferation and production of inflammation-associated cytokines, namely, IL-6 and TNF- $\alpha$. Moreover, its inhibitory effects on LPS-induced inflammation in macrophages were dependent on the NF- $\kappa \mathrm{B}$ signaling pathway. However, whether other key genes of this typical signaling pathway are involved in $\mathrm{m}^{6} \mathrm{~A}$ modification in macrophages except for $\mathrm{NF}-\kappa \mathrm{B}$ needs to be investigated by more future studies.

In summary, this study shows strong evidence supporting the vital role of METTL3 in RA. METTL3 can attenuate LPSinduced inflammation in macrophages through NF- $\kappa$ B. The findings in this study are useful for understanding RA pathogenesis and exploring novel biomarkers for RA diagnosis and treatment.

\section{Data Availability}

The data used to support the findings of this study are included within the article.

\section{Conflicts of Interest}

The authors declare that they have no competing interests.

\section{Authors' Contributions}

Jinghua Wang, Shushan Yan, and Hongying Lu are co-first authors.

\section{Acknowledgments}

This work was supported by grants from the National Natural Science Foundation (81601408), Shandong Natural Science Foundation for Young Scholars (ZR2019QH012 and ZR2016HQ12), and Weifang Science and Technology Development Program (2019GX031, 2019YX020, and 2017YX019).

\section{References}

[1] D. L. Scott, F. Wolfe, and T. W. Huizinga, "Rheumatoid arthritis," Lancet, vol. 376, no. 9746, pp. 1094-1108, 2010.

[2] D. Aletaha, T. Neogi, A. J. Silman et al., "2010 Rheumatoid arthritis classification criteria: an American College of Rheumatology/European League Against Rheumatism collaborative initiative," Arthritis and Rheumatism, vol. 62, no. 9, pp. 2569-2581, 2010.

[3] W. M. Jefferies, "The etiology of rheumatoid arthritis," Medical Hypotheses, vol. 51, no. 2, pp. 111-114, 1998.

[4] L. Klareskog, K. Lundberg, and V. Malmstrom, "Autoimmunity in rheumatoid arthritis: citrulline immunity and beyond," Advances in immunology, vol. 118, pp. 129-158, 2013.

[5] J. Suurmond, A. J. Schuerwegh, and R. E. Toes, "Anti-citrullinated protein antibodies in rheumatoid arthritis: a functional role for mast cells and basophils?," Annals of the Rheumatic Diseases, vol. 70, pp. 55-58, 2011.

[6] E. Perry, C. Kelly, P. Eggleton, A. De Soyza, and D. Hutchinson, "The lung in ACPA-positive rheumatoid arthritis: an initiating site of injury?," Rheumatology, vol. 53, no. 11, pp. 1940-1950, 2014.

[7] X. Wu, L. Sang, and Y. Gong, "N6-Methyladenine RNA modification and cancers," American journal of Cancer Research, vol. 8, no. 10, pp. 1957-1966, 2018.

[8] K. Hong, "Emerging function of N6-methyladenosine in cancer," Oncology Letters, vol. 16, no. 5, pp. 5519-5524, 2018.

[9] R. M. Rubio, D. P. Depledge, C. Bianco et al., "RNA m(6)A modification enzymes shape innate responses to DNA by regulating interferon beta," Genes \& Development, vol. 32, no. 2324, pp. 1472-1484, 2018.

[10] N. Lu, X. Li, J. Yu et al., "Curcumin attenuates lipopolysaccharide-induced hepatic lipid metabolism disorder by modification of m(6) A RNA methylation in piglets," Lipids, vol. 53, no. 1, pp. 53-63, 2018.

[11] X. Yin, R. Sachidanandam, S. Morshed, R. Latif, R. Shi, and T. F. Davies, "mRNA-Seq reveals novel molecular mechanisms and a robust fingerprint in Graves' disease," The Journal of Clinical Endocrinology and Metabolism, vol. 99, no. 10, pp. E2076-E2083, 2014.

[12] W. V. Gilbert and T. A. Bell, "Messenger RNA modifications: form, distribution, and function," Science, vol. 352, pp. 14081412, 2016.

[13] S. Geula, S. Moshitch-Moshkovitz, D. Dominissini et al., "Stem cells. m6A mRNA methylation facilitates resolution of naive pluripotency toward differentiation," Science, vol. 347, no. 6225 , pp. 1002-1006, 2015.

[14] K. D. Meyer, "Rethinking m6A readers, writers, and erasers," Annual Review of Cell and Development Biology, vol. 33, pp. 319-342, 2017.

[15] S. Geula, S. Moshitch-Moshkovitz, D. Dominissini et al., "Stem cells m6A mRNA methylation facilitates resolution of naïve 
pluripotency toward differentiation," Science, vol. 347, no. 6225, pp. 1002-1006, 2015.

[16] J. Liu, M. A. Eckert, B. T. Harada et al., "m(6)A mRNA methylation regulates AKT activity to promote the proliferation and tumorigenicity of endometrial cancer," Nature Cell Biology, vol. 20, no. 9, pp. 1074-1083, 2018.

[17] L. He, J. Li, X. Wang et al., "The dual role of N6methyladenosine modification of RNAs is involved in human cancers," Journal of Cellular and Molecular Medicine, vol. 22, no. 10, pp. 4630-4639, 2018.

[18] M. Chen, L. Wei, C. T. Law et al., "RNA N6-methyladenosine methyltransferase-like 3 promotes liver cancer progression through YTHDF2-dependent posttranscriptional silencing of SOCS2," Hepatology, vol. 67, no. 6, pp. 2254-2270, 2018.

[19] Z. Feng, Q. Li, R. Meng, B. Yi, and Q. Xu, "METTL3 regulates alternative splicing of MyD88 upon the lipopolysaccharideinduced inflammatory response in human dental pulp cells," Journal of Cellular and Molecular Medicine, vol. 22, no. 5, pp. 2558-2568, 2018.

[20] F. R, L. R, and S. S. J, "Review of head-to-head study designs in rheumatoid arthritis," Seminars in Arthritis and Rheumatism, vol. 46, no. 3, pp. 279-285, 2016.

[21] T. T. Glant, K. Mikecz, and T. A. Rauch, "Epigenetics in the pathogenesis of rheumatoid arthritis," BMC Medicine, vol. 12, p. 35, 2014.

[22] E. Karouzakis, R. E. Gay, S. Gay, and M. Neidhart, "Epigenetic deregulation in rheumatoid arthritis," Epigenetic Contributions in Autoimmune Disease, vol. 711, pp. 137-149, 2011.

[23] F. M. Brennan and I. B. McInnes, "Evidence that cytokines play a role in rheumatoid arthritis," The Journal of Clinical Investigation, vol. 118, no. 11, pp. 3537-3545, 2008.

[24] J. M. Adams and S. Cory, "Modified nucleosides and bizarre 5 'termini in mouse myeloma mRNA," Nature, vol. 255, no. 5503, pp. 28-33, 1975.

[25] Y. Furuichi, M. Morgan, A. J. Shatkin, W. Jelinek, M. SaldittGeorgieff, and J. E. Darnell, "Methylated, blocked 5 termini in HeLa cell mRNA," Proceedings of the National Academy of Sciences, vol. 72, no. 5, pp. 1904-1908, 1975.

[26] Y. Yang, P. J. Hsu, Y. S. Chen, and Y. G. Yang, "Dynamic transcriptomic m(6)A decoration: writers, erasers, readers and functions in RNA metabolism," Cell Research, vol. 28, no. 6, pp. 616-624, 2018.

[27] G. Jia, Y. Fu, and C. He, "Reversible RNA adenosine methylation in biological regulation," Trends in Genetics, vol. 29, no. 2, pp. 108-115, 2013.

[28] K. D. Meyer and S. R. Jaffrey, "The dynamic epitranscriptome: N6-methyladenosine and gene expression control," Nature Reviews Molecular Cell Biology, vol. 15, no. 5, pp. 313-326, 2014.

[29] J. A. Bokar, M. E. Shambaugh, D. Polayes, A. G. Matera, and F. M. Rottman, "Purification and cDNA cloning of the AdoMet-binding subunit of the human mRNA (N6-adenosine)-methyltransferase," RNA, vol. 3, no. 11, pp. 1233-1247, 1997.

[30] S. Lin, J. Choe, P. Du, R. Triboulet, and R. I. Gregory, “The $\mathrm{m}(6) \mathrm{A}$ methyltransferase METTL3 promotes translation in human cancer cells," Molecular Cell, vol. 62, no. 3, pp. 335345, 2016.

[31] Y. Du, G. Hou, H. Zhang et al., "SUMOylation of the m6ARNA methyltransferase METTL3 modulates its function," Nucleic Acids Research, vol. 46, no. 10, pp. 5195-5208, 2018.
[32] J. Tong, R. A. Flavell, and H. B. Li, "RNA m(6)A modification and its function in diseases," Frontiers of Medicine, vol. 12, no. 4, pp. 481-489, 2018.

[33] X. L. Ping, B. F. Sun, L. Wang et al., "Mammalian WTAP is a regulatory subunit of the RNA N6-methyladenosine methyltransferase," Cell Research, vol. 24, no. 2, pp. 177-189, 2014.

[34] S. Yan, G. Liu, C. Jin et al., "MicroRNA-6869-5p acts as a tumor suppressor via targeting TLR4/NF-kappaB signaling pathway in colorectal cancer," Journal of Cellular Physiology, vol. 233, no. 9, pp. 6660-6668, 2018.

[35] B. Hoesel and J. A. Schmid, "The complexity of NF- $\kappa$ B signaling in inflammation and cancer," Molecular Cancer, vol. 12, p. 86, 2013.

[36] S. C. Gupta, C. Sundaram, S. Reuter et al., "Inhibiting NF- $\kappa$ B activation by small molecules as a therapeutic strategy," Biochimica et Biophysica Acta (BBA) - Gene Regulatory Mechanisms, vol. 1799, no. 10-12, pp. 775-787, 2010.

[37] Y. Wang, F. Zheng, G. Gao et al., "MiR-548a-3p regulates inflammatory response via TLR4/NF- $\kappa$ B signaling pathway in rheumatoid arthritis," Journal of Cellular Biochemistry, vol. 120, pp. 1133-1140, 2018. 


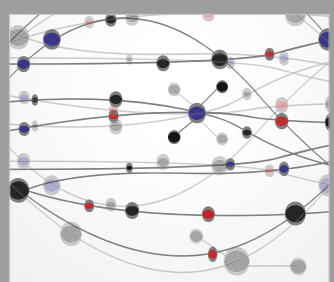

The Scientific World Journal
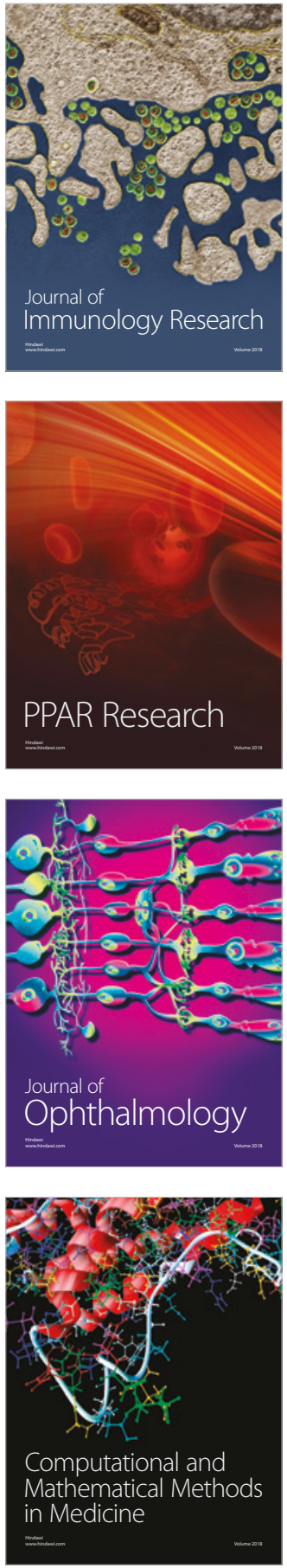

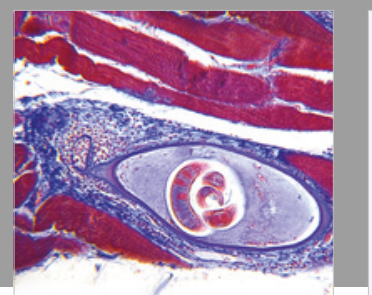

Gastroenterology Research and Practice

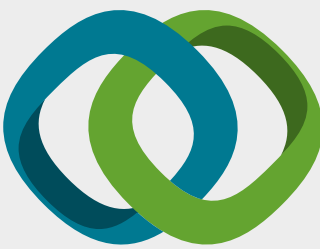

\section{Hindawi}

Submit your manuscripts at

www.hindawi.com
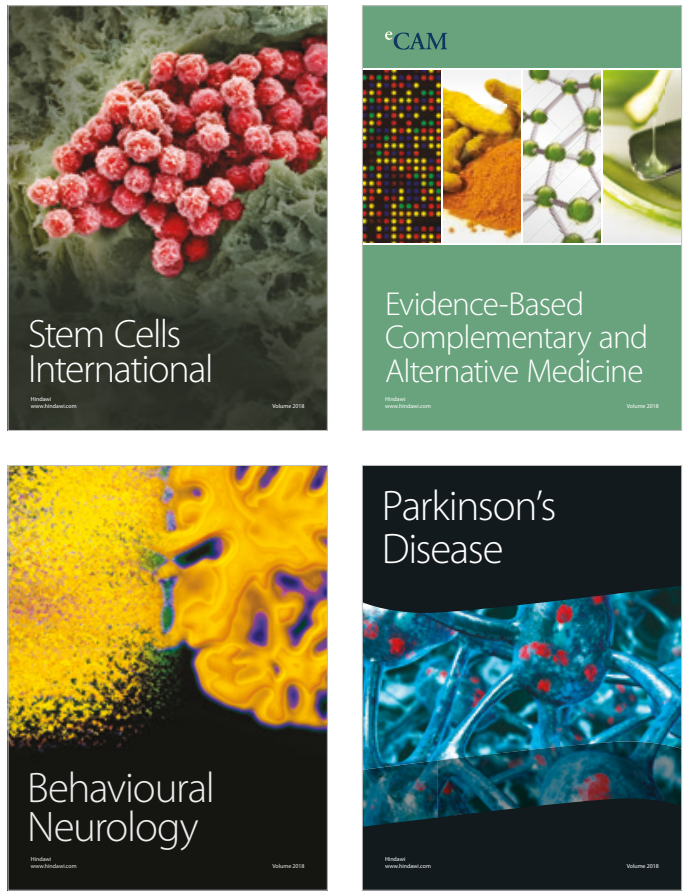

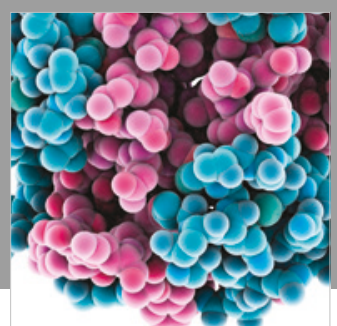

ournal of

Diabetes Research

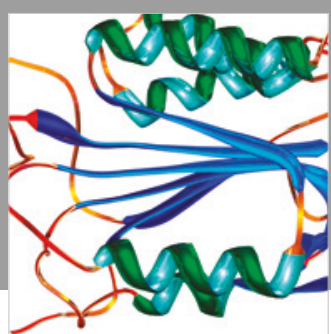

Disease Markers
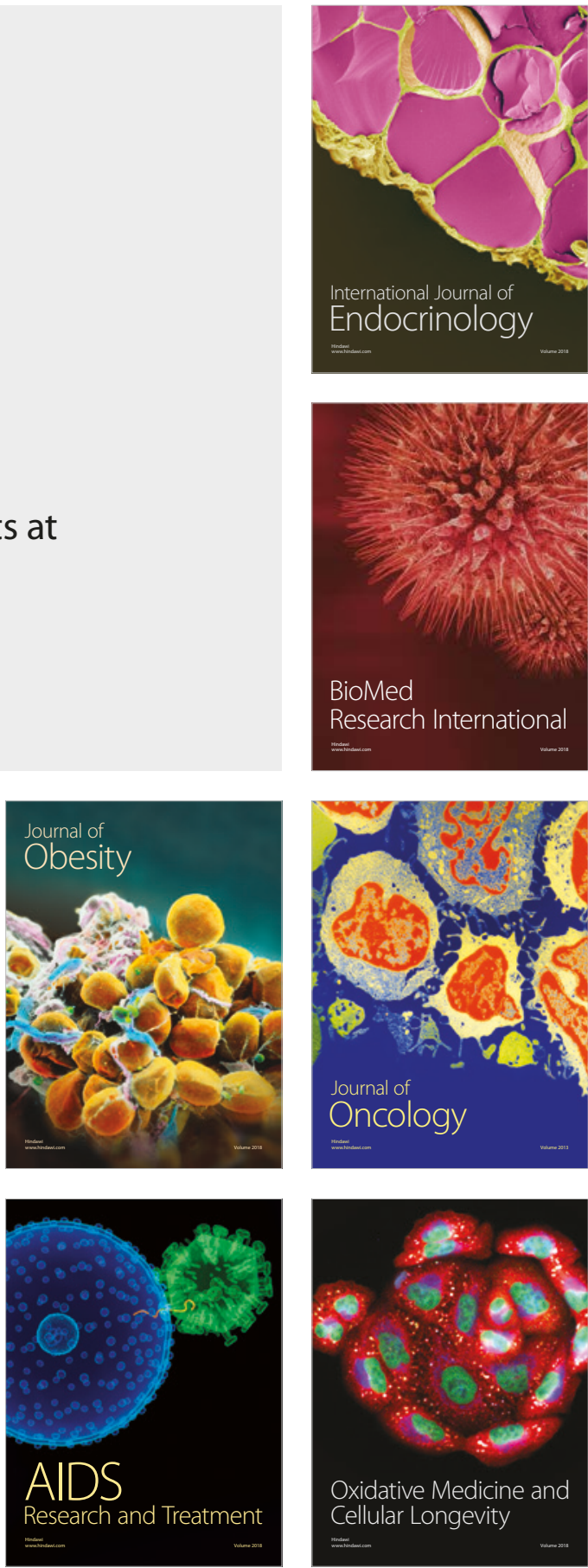